\title{
Framework Utilizing Machine Learning to Facilitate Gait Analysis as an Indicator of Vascular Dementia
}

\author{
Arshia Khan, Janna Madden \\ Department of Computer Science, University of Minnesota, \\ Duluth \\ Duluth, Minnesota, United States
}

\author{
Kristine Snyder \\ Department of Mathematics and Statistics \\ University of Minnesota, Duluth \\ Duluth, Minnesota, United States and Stryd, \\ Boulder, Colorado, United States
}

\begin{abstract}
Vascular dementia (VD), the second most common type of dementia, effects approximately 13.9 per cent of people over the age of 71 in the United States alone. $26 \%$ of individuals develop VD after being diagnosed with congestive heart failure. Memory and cognition are increasingly affected as dementia progresses. However, these are not the first symptoms to appear in some types of dementia. Alterations in gait and executive functioning have been associated Vascular Cognitive Impairment (VCI). Research findings suggest that gait may be one of the earliest affected systems during onset of VCI, immediately following a vascular episode. The diagnosis tools currently utilized for VD are focused on memory impairment, which is only observed in later stages of VD. Hence we are proposing a framework that isolates gait and executive functioning analysis by applying machine learning to predict VD before cognition is affected, so pharmacological treatments can be used to postpone the onset of cognitive impairment. Over a period of time, we hope to be able to develop prediction algorithms that will not only identify but also predict vascular dementia.
\end{abstract}

Keywords-Gait; machine learning; vascular dementia; early diagnosis; indicators; gait analysis

\section{INTRODUCTION}

The effects of vascular dementia are widespread. Currently, approximately $13.9 \%$ of individuals over the age of 71 have some form of dementia. As the demographics of our population change, the impact of chronic disease continues to increase. Currently, there are 35 million people in the United States over the age of 65. By 2030, this number is expected to be more than 70 million [1]. Vascular Cognitive impairment (VCI), the second widely spread type of dementia [2], second only to Alzheimer's Dementia (AD), occurs in $26 \%$ of individuals who are diagnosed with Congestive Heart Failure (CHF) [3]. Dementia is of particular concern as the decline of memory and cognition functioning lead to a loss of independence and increased dependency on families and healthcare systems [1]. The prevalence of Vascular Dementia $(\mathrm{VD})$, second to $(\mathrm{AD})$, doubles every 5.3 years, while the prevalence of $\mathrm{AD}$ doubles every 4.3 year. Just like $\mathrm{AD}$, the severity of VD is related to age. The age-adjusted rates of VCI are 14.6 per thousand per years, while it is 19.2 for AD [4]. Much of the healthcare costs incurred from dementia fall into the realm of government programs. However, individual caregivers also experience decrease in income and chronic fatigue for their efforts [1]. Early diagnosis of dementia, cannot stop the effect of the disease. While, it has been suggested that early treatment may slow the progression of the disease [5], developing an evaluation or monitoring protocol has proven challenging.

In this paper, we look at the background of subcortical vascular cognitive impairment, the use of technology in its diagnosis, typical onset patterns and early indicators. Following this a framework for applying machine learning techniques to these early indicators is presented. The paper concludes with a discussion on current techniques and future research direction.

\section{BACKGROUND}

Vascular dementia, the second most common type of dementia [6]-[9] was first identified by Thomas Willis in 1684 as a palsy that would cause 'stupidity' after stroke. Approximately $20-30 \%$ of individuals affected by stroke develop vascular dementia [2]. 36 million people around the world currently suffer from dementia. Vascular occlusions of larger vessels result in stroke, whereas the occlusion of smaller vessels results in a gradual degradation of subcortical areas of the brain that perform executive functions. This condition of small vessel occlusion is termed as Subcortical Vascular Dementia, (SVD), a most common type of VD [6], [8], [9]. 36-67\% of the vascular dementia is subcortical vascular dementia [10]. Our paper will focus on the SVD form of vascular dementia.

The use of technology as a tool in the medical setting is a growing area of research and development. With the desire to reduce healthcare costs and support patient-centered care, the integration of technology into medical processes is increasingly common [11]. At first these technologies were designed primarily for practitioners, managers, and other professionals in a healthcare setting. However, as telecommunication and mobile computing technologies improved, systems that interact directly with patients outside of the healthcare setting became more prevalent [12]. Perhaps the strongest force driving this innovation is the growing interest in evidence-based and personalized medicine. The origins of evidence-based medicine can be traced back to mid19th century Paris where physicians "conscientiously and explicitly" used outcomes from their previous cases to make current care decisions [13]. The rise of technology has greatly changed how evidence-based medicine is practiced, but the core idea has remained the same -- incorporating clinical 
evidence from systematic research into health decisions, particularly diagnosis [13]. Likewise, personalized medicine looks to base health decisions on individual health records, the idea being that individuals' patterns and health history should be incorporated into health decisions. Often referred to as $\mathrm{N}$ of-1 studies, mobile technology has proven to be of great utility in the execution of these studies to collect, record and communicate current data with providers [14].

Personalized medicine tools have been applied to many realms of health, including preventive care, chronic disease management, and monitoring of patients. Such systems allow us to ask previously unanswerable questions to better understand the connection between behavioral choices and health. There are many benefits to such a system. However, they come with many challenges. Because of the sheer mass of data, it is necessary to develop analytical methods to process raw data into actionable knowledge for patients and their providers [15]. In addition, regulatory, financial reimbursement, and technical security hurdles need to be considered.

This research looks at the potential of machine learning tools to analyze gait. Research has begun to recognize patterns in vascular dementia onset. However, in many cases, particular elements of gait are studied in isolation. We are proposing a framework that incorporates a larger number of measurable elements of gait and utilizes machine learning to optimize and improve upon past work.

\section{A. Progression of Vascular Dementia}

Progression of Vascular Dementia generally represents a stepwise decline - appearing suddenly after episode and aggravated from following episodes, but without the continuous decline common to Alzheimer's Disease. Vascular Dementia transitions from preclinical to Vascular Cognitive Impairment to Vascular Dementia can be sub-classified as mild, moderate or severe. To understand each diagnosis, the symptoms and expected progression at each of these stages must be considered.

1) Risk factors: The risk factors for vascular dementia are the same as cardiovascular diseases such as hypertention, stroke, atrial fibrilation, aortic fibrilation, diabetes mellitus type 2, obesity, lack of active lifestyle, depression, sleep apnia, and smoking [6]. In addition, the hemiparetic type of gait was identified as a risk factor [16].

2) Symptoms: Two of the foremost cognitive domains affected by vascular dementia are gait and executive functioning [17]. Among other cognitive domains affected, such as problem solving, execution of complex commands and motor skills, memory is the most significant [6].

3) Preclinical Stage: The initial stage is often described as "silent", as the brain begins to change without measureable symptoms being displayed. Changes are not detectable on tests and the symptoms patient experiences are not diagnosable. Because of this, much that is known about the preclinical stage of Vascular Dementia is based on retrospective evaluations of records of diagnosed cases. One such study found that patients had memory complaints 12 years prior to diagnosis and had experienced declines in activities of daily living 5 to 7 years previous to diagnosis [18]. While Vascular Dementia patients had memory complaints 12 years prior to diagnosis, cognitively, there is comparatively less deterioration in the preclinical stage as compared to other forms of Dementia. Patients' with incident vascular dementia deteriorate earlier and faster in daily functioning, especially the more physical activities of daily living such as activities, arising, dressing and grooming, eating, hygiene, grip, reach, and walking, as compared to other forms of Dementia that experience the first changes in cognitive activities such as finance management, phoning, medication use, housekeeping, and meal preparation [18], [19]. In addition, the preclinical stage is often accompanied by symptoms of depression, particularly motivation-related such as lack of interest, loss of energy and concentration difficulties. This association still remained significant after adjusting for memory complaints, showing that depressive symptoms are not merely a by-product of perceived cognitive difficulties [20].

4) Vascular Cognitive Impairment: The progression from preclinical to Vascular Cognitive Impairment is not an unambiguous transition. The Vascular Cognitive Impairment stage is loosely defined as cases where one or more cognitive domains becomes significantly affected [21], [22]. At this stage in the disease, symptoms are becoming clinically detectable and while noticeable in daily living, they are not generally too limiting in this respect.

5) Vascular Dementia: Onset of Vascular Dementia is marked by cognitive impairment severe enough to interfere with everyday activities. The onset of Vascular Dementia can be divided into sub-domains of mild, moderate, moderately severe, and severe.

6) Mixed Dementia: Another factor to consider is the onset of other forms of cognitive impairment in addition to vascular dementia, referred to as "Mixed Dementia". Mixed Dementia refers to cases of Vascular Dementia where symptoms of other cognitive impairment, not originating from the vascular episode, begin to affect the patient in addition to the symptoms of Vascular Dementia already present. Approximately 15 percent of cases of Vascular Dementia present with other forms of cognitive impairment [23]. Identifying the onset of other cognitive impairments and the relationship between the Vascular episode and these other cognitive impairments is one of the great challenges facing research in this area.

\section{B. Gait as an early Indicator of Vascular Dementia}

The challenge of diagnosing Vascular Dementia during or prior to vascular cognitive impairment is one that much research has looked into. Vascular dementia in particular is of interest because patients often experience a "step-wise" deterioration as opposed to gradual and symptomatic decline following acute events. In addition, in the subcategory of vascular dementia, mild cognitive impairment seems equally if not more prevalent than full onset of dementia symptoms [24]. 
Researchers have identified several indicators of vascular cognitive impairment including impaired social or occupational functioning, motor activities, visual processing and abstract reasoning [24]. However, one challenge is to transform these rather abstract indicators into quantifiable and actionable metrics.

One developing area is that of gait analysis. This could be stride length, lateral balance, or effort exerted (measured using heart-rate monitor for example) for a particular class of activity [24]. Motor activity metrics focus on measuring ease, frequency, and type of movement. Gait has clear links to motor activities, but it also has an interesting link to visual processing since the visual system is strongly correlated with balance. In comparison to other physiological feedback systems, Visual information is typically more sensitive and is believed to play a significant role in fine-grained adjustments to balance, especially in the feet and ankles [25]. In patients with Vascular Dementia onset, impaired visual processing could be recognized in balance and gait discrepancies. Various gait metrics have been investigated, and their potential to identify vascular cognitive impairment has been evaluated.

1) Distance and Speed Metrics: In respect to gait analysis, distance and speed are typically measured in terms of a single stride. To understand what these metrics quantify, it's important to understand the components of a stride. Stride length is measured as the distance between two consecutive footfalls of the same foot. In addition, a single stride can be broken down into components, commonly, swing time and stance time. Swing time refers the time when only one foot is on the ground. The stance time refers to the time when that foot is on the ground. Distance and speed metrics are often in reference to either a complete stride or a component of a stride [26]. Shorter stride length has been linked to a increased chance of mild cognitive impairment [25]. Similarly, the a slower gait can indicate motor function concerns, thus measuring the velocity or stride frequency (steps per minute) can also provide useful information [26].

2) Gait Disturbances and Difficulties: In a similar vein, measuring gait disturbances or difficulties takes stride metrics and looks for abnormalities or inconsistencies between multiple instances over a given time period. In these methods, a range of acceptable metrics is defined and measurements outside that range are considered a disturbance [27]. This idea comes up frequently in fall detection, but the idea of creating a customized range based on user data rather than setting a predefined range, is one application of this idea that may prove useful to this research.

3) Pace, Rhythm, and Variability of Gait: Pace, rhythm, and variability of gait also look at comparing multiple strides in a time frame against each other. However, rather than looking for outliers, these metrics attempt to recognize patterns in the data and cases where these patterns are not true [27]. One example of this is measuring the percentage of the stride that is spent on the swing verses the percent on the stance. Typically, the swing takes 40 percent of the stride with stance taking the remaining 60 percent of the time [28]. Pace, rhythm, and variability measures are aimed to quantify how much a gait changes in this respect over a time period.

4) Stability: Stability and width of base, while associated with pace, are largely metrics of balance. Stability looks at the consistency of weight patterns on a foot. Inconsistent (variable) patterns of weight distribution could indicate the individual is "shaky" and struggling to balance. Width of base is another way we can measure stability. Width of base refers to how far apart a individual's stance is. A wide stance can indicate balance concerns as well [28]. Finally, double support, when both feet are in contact with the ground at the same time, can be measured while an individual is walking. Longer double support time, can also be correlated to balance [26].

5) Outstanding Challenges in Gait Analysis: A challenge facing this realm of research is that aging causes many of these occurrences. Therefore successful research in this field has looked not only for the presence of these indicators, most of which are likely common in an aging population, but also at the extent to which an indicator is present. This aims to separate changes typical of aging from those of dementia onset. However, this continues to be a challenge in this field of study [24], [25]. In addition, since the indicators at this stage are largely behavioral and physiological it is very challenging to identify these in a clinical setting. In addition, the way these indicators present in each patient differs, and, in many cases, a patient may experience only a subset of all the indicators mentioned. Adjusting for individual differences and variation during analysis is another challenge of this field.

\section{Sensors for Collecting Gait Measures}

As discussed above, there are many different metrics we can use to describe gait, including distance walked, speed, disturbances or difficulties, pace, rhythm, variability, stability and width of base. Most research conducted on gait analysis in the clinical setting relies on health care providers observing and manually identifying the presence or prevalence of certain features [29]. We have the technological capabilities to streamline this into a single process. There are three primary classes of technology being used in gait analysis: pressurebased, force-based, and mobile device sensors.

Pressure-based and force-based systems have been developed by numerous groups as a way to monitor gait. These sensors are typically designed as an insole with multiple pressure sensors throughout the insole or a treadmill that can identify how much weight is being exerted in a location at a particular time. From this, center of mass and the elements of pace and stability can be derived. In addition, these systems excel at identifying stability, balance, and the base stance [30]. For example, such a system could determine which part of the foot bears most of the weight of the individual when flat footed or if when they are motionless, there is a continuous shift to where their center of mass is, indicating issues maintaining a balanced stance.

The other type of device that is showing great potential in the area of gait detection is mobile device sensors. Mobile devices include a multitude of sensors, including an 
accelerometer, digital compass, gyroscope, proximity sensor, ambient light sensor, GPS, microphone, and camera [31]. Using these sensors, a device can be configured to track elements of gait such as stride length, base width, pace, and variability, as well as distance travelled and speed travelled [32]. In addition, applications can use activity recognition algorithms on the device to $\log$ data only when the desired activity is present, which improves the specificity of the data being collected.

As can be seen, there is much overlap between the capabilities of pressure-based insole sensors and mobile device sensors. However, a few aspects set them apart. Pressure-based systems lend themselves to tracking balance and stability better than mobile devices, while mobile devices benefit from increased variability of sensors and computing power that makes them capable of more powerful aggregations, measuring speed, distance, and classifying motion.

\section{Machine Learning}

The problem of diagnosing vascular dementia early in onset is a challenging one. For one, not all symptom patterns or indicators will be present in an individual patient, but rather a subset of these. The ideal system would have to be flexible enough to account for the variability in disease onset, while also not being so flexible as to create a multitude of falsepositive results. Another challenge is that behavioral and physiological patterns differ between individuals. To account for this, the system must learn to confirm the individuality of measures to the known pattern, to create personalized and actionable knowledge. There are there are numerous learning models to explore and within each model, multiple attributes that affect the effectiveness of the model. When choosing models to test, we are looking for a two-part classifier. However, the problem is complicated by the fact that not all vascular dementia onsets in the same way. For this reason, the way attributes interrelate will be an important focus of an effective model.

\section{METHODOLOGY}

Our study focuses on developing a framework for identification and prediction of subcortical vascular dementia. The framework involves analyzing the gait and executive functioning using machine learning. This methodology involves two parts. Part one focuses on gait analysis while part two focuses on executive functioning evaluation as seen in Fig. 4.

Phase 1: Gait Analysis: Our framework proposes force plate gait measurement system that utilizes Piezoelectric measurement technology for accurate force and moment data gathering. Measuring changes in gait pattern or alterations in the center of mass enables tracking the changes in gait after a significant cardiovascular event. Gait analysis can be traced back to the early eighties where the analysis was done to in the assessment of cerebral palsy, neuromuscular disorders and other orthopedic evaluations [33].

Gait, a means of locomotion, is the style or manner of walking that can be measured by two domains of measurement systems: i) Spatiotemporal system-where the step length, step width, speed and stride frequency are taken into consideration; and ii) Kinematic -where the angles of joints and rotation of knee, hip and ankle are taken into consideration. Some of the parameters associated with gait that are valuable in the prediction of VD are velocity, center of gravity, stride frequency, step length and frequency, and symmetry of limb movement, defined and depicted in Table II and Fig. 1, respectively [7], [34]. In particular, VD is predictive by the following specific characteristics of gait as seen in Table I [34]. The technique for calculating these attributes of motion can be seen in in Fig. 2 and 3.

TABLE I. GAIT PARAMETERS IN VASCULAR DEMENTIA

\begin{tabular}{|l|l|l|}
\hline$\#$ & Parameter & Variation in VD \\
\hline 1 & Velocity & Slower \\
\hline 2 & Stride length & Shorter \\
\hline 3 & Posture/Balance & Instability \\
\hline 4 & Start & Hesitation/Freezing \\
\hline 5 & Stride Frequency & Decreased \\
\hline 6 & Gait lines variability & Increased \\
\hline 7 & Tandem gait & Increased \\
\hline 8 & Arm swing & Decreased \\
\hline
\end{tabular}

TABLE II. DEFINITION OF QUANTITATIVE GAIT PARAMETERS. ALL QUANTITATIVE PARAMETERS DESCRIBED BELOW ARE AUTOMATICALLY CALCULATED AS THE MEAN OF TWO TRIALS BY THE GAIT SOFTWARE [2], [26], [34]

\begin{tabular}{|l|l|}
\hline Variable & Description \\
\hline Velocity & Distance covered over a period of time \\
\hline Stride length & $\begin{array}{l}\text { Distance between heel points of two steps of the } \\
\text { same foot }\end{array}$ \\
\hline Stride Frequency & Steps per unit of time \\
\hline Balance/Posture & Center of gravity variation \\
\hline
\end{tabular}
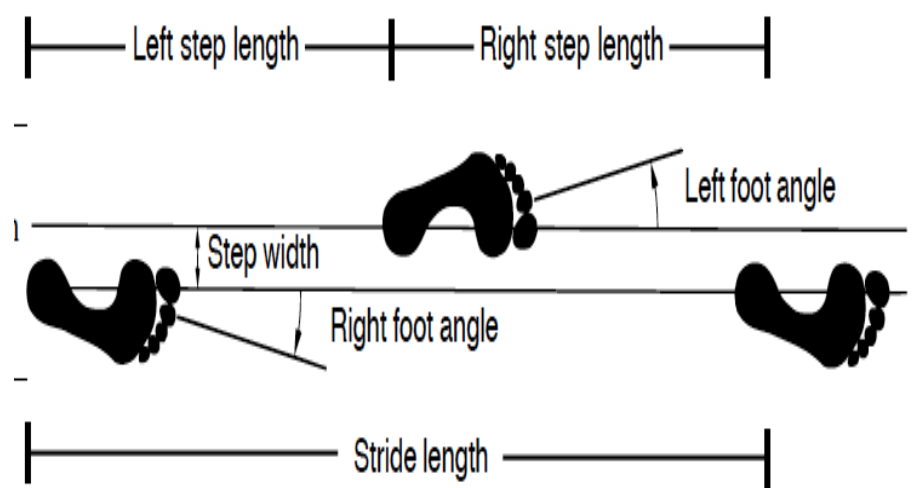

Fig. 1. Step and Stride in Gait: Adapted from [2]. 


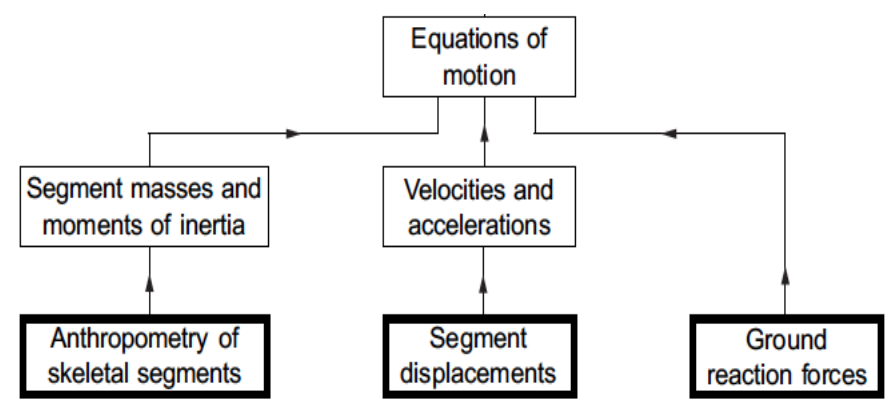

Fig. 2. Gait Parameters and Motion in Words: Adopted from [2].

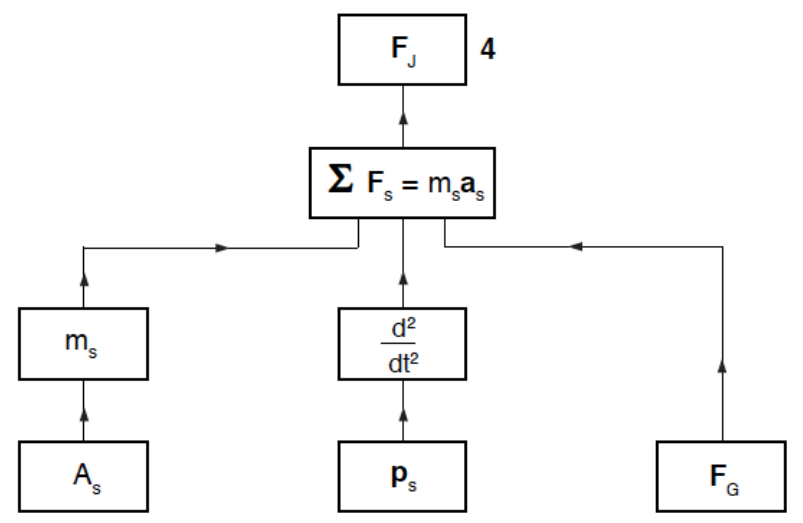

Fig. 3. Formulas for Gait Parameters and Motion: Adopted from [2].

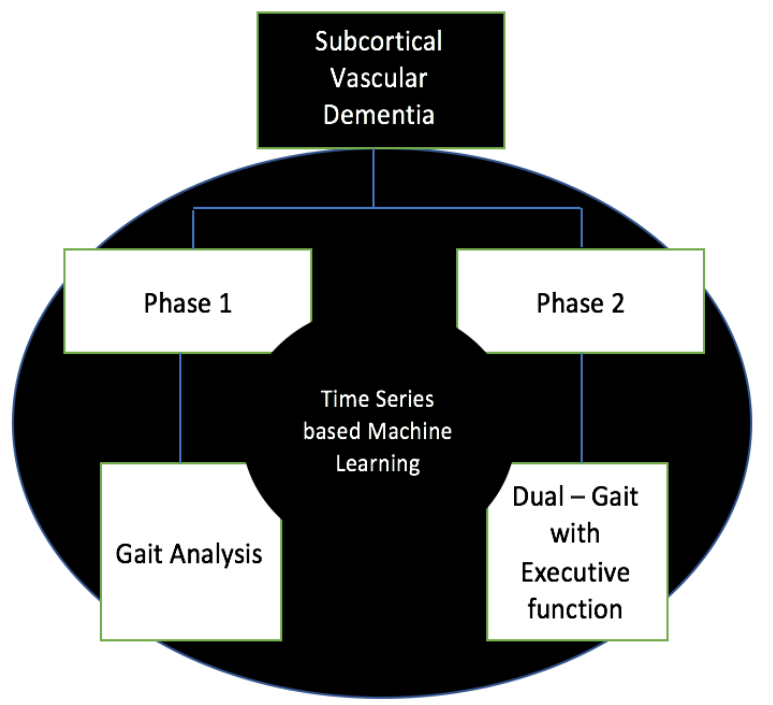

Fig. 4. Framework Combining Gait and Executive Function Analysis Applying Time Series based Machine Learning.

Phase 2: Executive function Analysis: Dual task performance in gait has been recognized as a marker for VD [35], [37]. In the second part of the protocol, the framework performs executive function analysis by having the subject walk and recognizes verbs and walk and finger tap. The evaluation will be performed in two steps:

\section{Step 1: The subject will walk and finger tap}

Step 2: The subject will walk and recognize verbs from pictures that are projected on the wall facing the walk.
The Stride length and gait speed will be tracked during this phase, as executive function disorders were recognized as a reduction in gait speed and stride length [33]. One mechanism for examining executive function is by verb recognition or by dual function performance such as finger tapping while walking or verb recognition while walking [33], [35]-[37].

\section{DISCUSSION}

A combination of alterations in gait and executive functioning has been identified as a 5 year predictor for VD [8], [9], [16], [38]. Our proposal addresses subcortical vascular dementia that affects gait and executive functioning in affected individuals [17]. Although vascular dementia is marked by its impact on problem solving, execution of complex commands, motor skills, and memory, these domains are not the first to be noticed. VD first impacts gait and executive functioning, and hence we are proposing a framework that uses machine learning to identify alterations in gait and executive functioning [17]. According to the National Institute of Health and Care Excellence (NICE) guidelines the diagnosis of vascular dementia is performed by tools that are based on memory impairment but the impairment is observed only in later stages of VD. Hence we are proposing a system that would diagnose VD earlier than the initiation of the impairment so pharmacological treatments can be utilized to help postpone cognitive impairment. Tests such as minimental state examination (MMSE) do not test for executive functioning impairment, which renders them insignificant tools for Vascular dementia identification. Although Montreal Cognitive Assessment (MOCA) does test for executive functioning impairment and can be used for VD, it relies on factors that can be identified only in later stages of dementia hence reducing its value in treatment of memory impairment. There is a need for tools that can identify and predict VD before its markers are evident. These tools will help clinicians treat individuals with pharmacological remedies that can help minimize cognition impairments as a result of VD.

\section{CONCLUSION}

The uniqueness of our approach is the application of machine learning along with a combination of gait and executive function analysis. Several research studies have identified gait as an important marker in early identification of vascular dementia [8], [9], [16], [38], while some other researchers have identified executive function as an early marker for subcortical vascular dementia [33], [35]-[37]. We are proposing a framework that combines gait and executive function as a hybrid early predictor of subcortical vascular dementia. The parameters for gait such as reduction in velocity, stride frequency, stride length, and dual cognitive functioning such as finger tapping while walking and verb recognition while walking, with time series based machine learning analysis.

Future work should be focused on implementing a machine learning technique that combines gait and executive functioning metrics utilizing this framework. In addition, research into incorporating other early indicators of subcortical vascular dementia into the framework should be pursued. 


\section{REFERENCES}

[1] Plassman, Brenda L., et al. "Prevalence of dementia in the United States: the aging, demographics, and memory study." Neuroepidemiology 29.12 (2007): 125-132.

[2] Vaughan, Christopher L., Brian L. Davis, and C. O. Jeremy. "Dynamics of human gait." (1999).

[3] Román, Gustavo C. "Vascular dementia may be the most common form of dementia in the elderly." Journal of the neurological sciences 203 (2002): 7-10.

[4] Gorelick, Philip B. et al. "Vascular Contributions to Cognitive Impairment and Dementia: A Statement for Healthcare Professionals From the American Heart Association/American Stroke Association." Stroke; a journal of cerebral circulation 42.9 (2011): 2672-2713. PMC. Web. 5 Apr. 2018.

[5] Petersen, Ronald C., et al. "Practice parameter: Early detection of dementia: Mild cognitive impairment (an evidence-based review) Report of the Quality Standards Subcommittee of the American Academy of Neurology." Neurology 56.9 (2001): 1133-1142.

[6] Bonnici-Mallia, Anne M., Christopher Barbara, and Rahul Rao. "Vascular cognitive impairment and vascular dementia." InnovAiT (2018): 1755738018760649.

[7] Beauchet, Olivier et al. "Guidelines for Assessment of Gait and Reference Values for Spatiotemporal Gait Parameters in Older Adults: The Biomathics and Canadian Gait Consortiums Initiative." Frontiers in Human Neuroscience 11 (2017): 353. PMC. Web. 13 Apr. 2018.

[8] Cummings, Jeffrey L. "Vascular subcortical dementias: clinical aspects." Dementia and Geriatric Cognitive Disorders 5.3-4 (1994): 177-180.

[9] Román, Gustavo C., et al. "Vascular dementia Diagnostic criteria for research studies: report of the NINDS-AIREN International Workshop." Neurology 43.2 (1993): 250-250.

[10] Román, Gustavo C., et al. "Subcortical ischaemic vascular dementia." The Lancet Neurology 1.7 (2002): 426-436

[11] P. Chowriappa, S. Dua, and Y. Todorov. "Introduction to machine learning in healthcare informatics". Machine Learning in Healthcare Informatics. Springer, 2014, pp. 1-23.

[12] G. Eysenbach. "Recent advances: Consumer health informatics". BMJ: British Medical Journal 320.7251 (2000), p. 171.

[13] D. L. Sackett. "Evidence-based medicine". Seminars in perinatology. Vol. 21. 1. Elsevier. 1997, pp. 3-5.

[14] E. O. Lillie, B. Patay, J. Diamant, B. Issell, E. J. Topol, and N. J. Schork. "The n-of-1 clinical trial: the ultimate strategy for individualizing medicine?" Personalized medicine 8.2 (2011), pp. 161173.

[15] M. Panahiazar, V. Taslimitehrani, A. Jadhav, and J. Pathak. "Empowering personalized medicine with big data and semantic web technology: promises, challenges, and use cases". Big Data (Big Data), 2014 IEEE International Conference on . IEEE. 2014, pp. 790795

[16] Verghese J, Derby C, Lipton R. High risk neurological gaits and vascular dementia. Neurology. 2006;66(suppl 2):A57A58.

[17] V. J. A. Verlinden, J. N. van der Geest, R. F. A. G. de Bruijn, A. Hofman, P. J. Koudstaal, and M. A. Ikram. "Trajectories of decline in cognition and daily functioning in preclinical dementia". Alzheimer's \& Dementia 12.2 (Feb. 1, 2016), pp. 144-153. issn: 1552-5260. doi: 10.1016/j.jalz.2015.

[18] R. A. Sperling et al. "Toward defining the preclinical stages of Alzheimer's Disease: Recommendations from the National Institute on Aging-Alzheimer's Association workgroups on diagnostic guidelines for Alzheimer's disease". Alzheimer's \& Dementia 7.3 (May 1, 2011), pp. 280-292. issn: 1552-5260. doi: 10.1016/j.jalz.2011.03.003.

[19] Berger, A-K., et al. "The occurrence of depressive symptoms in the preclinical phase of AD A population-based study." Neurology 53.9 (1999): 1998-1998.
[20] S. Gauthier, B. Reisberg, M. Zaudig, R. C. Petersen, K. Ritchie, K. Broich, S. Belleville, H. Brodaty, D. Bennett, H. Chertkow, J. L. Cummings, M. deLeon, H. Feldman, M. Ganguli, H. Hampel, P. Scheltens, M. C. Tierney, P.Whitehouse, and B. Winblad. "Mild cognitive impairment". The Lancet 367.9518 (Apr. 15, 2006), pp. 1262-1270. issn: 0140-6736. doi: 10.1016/S0140-6736(06)68542-5.

[21] B. C. Stephan, F. E. Matthews, K.-T. Khaw, C. Dufouil, and C. Brayne. "Beyond mild cognitive impairment: vascular cognitive impairment, no dementia(VCIND)". Alzheimer's Research \& Therapy 1.1 (July 9, 2009), p. 4. issn: 1758-9193. doi: 10.1186/alzrt4.

[22] K. Rockwood, C. Macknight, C. Wentzel, S. Black, R. Bouchard, S. Gauthier, H. Feldman, D. Hogan, A. Kertesz, and P. Montgomery. "The diagnosis of mixed dementia in the Consortium for the Investigation of Vascular Impairment of Cognition (CIVIC)". Annals of the New York Academy of Sciences 903.1 (2000), pp. 522-528.

[23] D. S. Knopman. "The initial recognition and diagnosis of dementia". The American journal of medicine 104.4 (1998), 2S-12.

[24] Hausdorff, Jeffrey M., and Aron S. Buchman. "What links gait speed and MCI with dementia? A fresh look at the association between motor and cognitive function." (2013): 409-411.

[25] J. Verghese, C. Wang, R. B. Lipton, R. Holtzer, and X. Xue. "Quantitative gait dysfunction and risk of cognitive decline and dementia”. Journal of Neurology, Neurosurgery \& Psychiatry 78.9 (2007), pp. 929-935.

[26] J. Verghese, R. B. Lipton, C. B. Hall, G. Kuslansky, M. J. Katz, and H. Buschke. "Abnormality of gait as a predictor of non-Alzheimer's dementia". New England Journal of Medicine 347.22 (2002), pp. 1761176.

[27] M. Heydarzadeh, C.-T. Tan, M. Nourani, and S. Ostadabbas. "Gait variability assessment in neuro-degenerative patients by measuring complexity of independent sources". Engineering in Medicine and Biology Society (EMBC), 2017 39th Annual International Conference of the IEEE. IEEE. 2017, pp. 3186-3189.

[28] T. IJmker and C. J. Lamoth. "Gait and cognition: the relationship between gait stability and variability with executive function in persons with and without dementia". Gait \& posture 35.1 (2012), pp. 126-130.

[29] K. Kong and M. Tomizuka. "A Gait Monitoring System Based on Air Pressure Sensors Embedded in a Shoe". IEEE/ASME Transactions on Mechatronics 14.3 (June 2009), pp. 358-370. issn: 1083-4435. doi: 10.1109/TMECH.2008.2008803

[30] N. D. Lane, E. Miluzzo, H. Lu, D. Peebles, T. Choudhury, and A. T. Campbell. "A survey of mobile phone sensing". IEEE Communications magazine 48.9 (2010).

[31] J. Juen, Q. Cheng, V. Prieto-Centurion, J. A. Krishnan, and B. Schatz. "Health monitors for chronic disease by gait analysis with mobile phones". Telemedicine and e-Health 20.11 (2014), pp. 1035-1041.

[32] Davis III, Roy B., et al. "A gait analysis data collection and reduction technique." Human movement science 10.5 (1991): 575-587.

[33] Morgan, Debra, et al. "The potential of gait analysis to contribute to differential diagnosis of early stage dementia: Current research and future directions." Canadian Journal on Aging/La Revue canadienne du vieillissement 26.1 (2007): 19-32.

[34] Beauchet, Olivier et al. "Gait Analysis in Demented Subjects: Interests and Perspectives." Neuropsychiatric Disease and Treatment 4.1 (2008): 155-160. Print.

[35] Piatt, Andrea L., et al. "Action (verb naming) fluency as an executive function measure: convergent and divergent evidence of validity." Neuropsychologia 37.13 (1999): 1499-1503.

[36] Salthouse, Timothy A. "Relations between cognitive abilities and measures of executive functioning." Neuropsychology19.4 (2005): 532.

[37] Foster, Jordana Bieze. "Neurologic Gait Predicts Vascular Dementia." Applied Neurology, 2006, p. 23.

[38] Allali, Gilles, Marian Van Der Meulen, and Frédéric Assal. "Gait and cognition: The impact of executive function." Swiss Archives of Neurology and Psychiatry 161.6 (2010): 195-199. 